\title{
Dynamics of propylene glycol and its oligomers confined to a single molecular layer
}

\author{
J. Swenson ${ }^{\text {a) }}$ and D. Engberg \\ Department of Applied Physics, Chalmers University of Technology, SE-412 96 Göteborg, Sweden \\ W. S. Howells \\ Rutherford-Appleton Laboratory, Chilton, Didcot, OX11 OQX, United Kingdom \\ T. Seydel \\ Institut Laue-Langevin, BP 156, F-38042 Grenoble Cedex 9, France \\ F. Juranyi \\ Paul Scherrer Institute, Neutron Scattering Laboratory, CH-5232 Villigen, Switzerland \\ and University of Saarland, D-66123 Saarbrucken, Germany
}

(Received 6 December 2004; accepted 4 May 2005; published online 24 June 2005)

\begin{abstract}
The dynamics of propylene glycol (PG) and its oligomers 7-PG and poly-propylene glycol (PPG), with $M_{w}=4000(\approx 70$ monomers), confined in a Na-vermiculite clay have been investigated by quasielastic neutron scattering. The liquids are confined to single molecular layers between clay platelets, giving a true two-dimensional liquid. Data from three different spectrometers of different resolutions were Fourier transformed to $S(Q, t)$ and combined to give an extended dynamical time range of $0.3-2000 \mathrm{ps}$. An attempt was made to distinguish the diffusive motion from the methyl group rotation and a fast local motion of hydrogen in the polymer backbone. The results show that the average relaxation time $\left\langle\tau_{d}\right\rangle$ of this diffusive process is, as expected, larger than the relaxation time $\langle\tau\rangle$ averaged over all dynamical processes observed in the experimental time window. More interesting, it is evident that the severe confinement has a relatively small effect on $\left\langle\tau_{d}\right\rangle$ at $T$ $=300 \mathrm{~K}$, this holds particularly for the longest oligomer, PPG. The most significant difference is that the chain-length dependence of $\left\langle\tau_{d}\right\rangle$ is weaker for the confined liquids, although the slowing down in bulk PG due to the formation of a three-dimensional network of $\mathrm{OH}$-bonded end groups reduces this difference. The estimated average relaxation time $\langle\tau\rangle$ at $Q=0.92 \AA^{-1}$ for all the observed processes is in excellent agreement with the previously reported dielectric $\alpha$ relaxation time in the studied temperature range of $260-380 \mathrm{~K}$. The average relaxation time $\langle\tau\rangle$ (as well as the dielectric $\alpha$ relaxation time) is also almost unaffected by the confinement to a single molecular layer, suggesting that the interaction with the clay surfaces is weak and that the reduced dimensionality has only a weak influence on the time scale of all the dynamical processes observed in this study.

(C) 2005 American Institute of Physics. [DOI: 10.1063/1.1943408]
\end{abstract}

\section{INTRODUCTION}

The structure and dynamics of molecular liquids and polymers have been studied widely for decades. More recently, there is a growing interest in thin polymer films due to their many technological applications in areas such as microelectronics, biomaterials, and medicine, where, e.g., the polymers are used to achieve certain desired surface properties. An increased understanding, on the microscopic level, of polymers at surfaces and in confined geometries would thus greatly assist in the development and improvement of new technologies. Furthermore, the structure and dynamics of polymers confined in a true two-dimensional geometry (such as the Na-vermiculite clay used here) are also interesting from a theoretical point of view since they present an opportunity to test several models and theoretical predictions for systems with a reduced dimensionality.

Despite comprehensive studies ${ }^{1-38}$ of the structure and

${ }^{a)}$ Electronic mail: f5xjs@fy.chalmers.se dynamics of liquids and polymers confined in various host materials it has been difficult to reach consensus on how pure geometrical confinement and surface interactions influence the system properties. The present $\mathrm{Na}-\mathrm{vermiculite}$ clay has been used as host material in some of these studies, ${ }^{1-6}$ similar clays have also been used, ${ }^{7-14}$ but in the majority of the investigations porous glasses have been used to confine the liquids and polymers in pores with a size of $2-10 \mathrm{~nm}$. In many of these studies the experimental conditions have appeared to be rather similar, but nevertheless the results often led to contradictory conclusions. Therefore, some authors ${ }^{18-20}$ propose that the pure geometrical confinement effect slows down the dynamics, whereas others ${ }^{22-29}$ have proposed that geometrical confinements always speed up the dynamics and that only surface interactions can give rise to slower relaxational motions compared to the corresponding bulk system. In the case of polymers intercalated in clays the latter opinion has been supported by a simultaneous slowing down of polymer segments which interact with the clay sur- 
faces and a speeding up of other polymer segments in the center of approximately 20 - $\AA$-thick polymer layers. ${ }^{9,10}$

Possible explanations for the apparently contradictory results in the literature are reviewed by McKenna in Ref. 39. One of these explanations, as was also discussed in more detail by Huwe et al., ${ }^{27}$ is that the density of the confined liquid is a key parameter, since it is well known from numerous pressure studies that the relaxation rate decreases with increasing density. Furthermore, Morineau et al. ${ }^{38}$ have shown that the density of liquids confined in crystalline porous materials can be experimentally determined with great accuracy. Thus, if such measurements show that the geometrical confinement gives rise to a reduced density compared to the bulk liquid the dynamics is expected to be accelerated, whereas the opposite behavior might be the case if the density should be increased. The importance of having a low density for fast dynamics was also pointed out by Manias et al. ${ }^{10}$ who showed that the dynamics was strongly enhanced in regions of low density. Other possibilities for the many diverging results are that the structural and dynamical properties are extremely sensitive to a wide range of parameters, such as the size and geometry (e.g., the dimension) of the confined space, the roughness of the surrounding walls, and the nature of the surface interactions (e.g., hydrophilic or hydrophobic). The influence of the different parameters may also be strongly temperature dependent, which makes the whole scenario very complicated, as discussed in Ref. 39.

In this study propylene glycol (PG), its 7-mer (7-PG), and poly-propylene glycol (PPG) with $M_{w}=4000 \quad(\approx 70$ monomers) were confined in the two-dimensional (2D) galleries of a $\mathrm{Na}$-vermiculite clay to obtain ultrathin layers of the liquids. The thickness of the liquid layers was only $5.5 \AA$ in the case of PG and $3.7 \AA$ for $7-\mathrm{PG}$ and $\mathrm{PPG}^{3}{ }^{3}$ In order to achieve the latter thickness the oligomers must form a flat monolayer with their methyl groups pointing in the direction of the clay surfaces. ${ }^{2}$ The slightly larger layer thickness for the monomers indicates that they should be orientated either perpendicular to the clay layers or parallel to the layers, but with the methyl groups pointing towards a clay surface. ${ }^{2}$ Since each macroscopically sized sample consists of nearly a million of such ultrathin parallel layers the vermiculite clay is ideal for producing truly two-dimensional liquids and polymers which are then possible to investigate with conventional experimental techniques. However, there are drawbacks with the naturally occurring vermiculite clays. Their inhomogeneous distribution of different ions makes it often difficult to obtain homogeneous layers of the intercalated liquids. In fact, for PPG and similar liquids it was evident from diffraction experiments that some of the interplatelet layers were not filled at all. ${ }^{3,5}$ This inhomogeneous distribution of the molecules resulted in considerably lower macroscopic average densities [estimated from the long time limit of the $S(Q, t)$ presented in Fig. 2(b) to be approximately $0.33,0.40$, and $0.50 \mathrm{~g} / \mathrm{cm}^{3}$ for PG, 7-PG, and PPG, respectively] and further made it difficult to estimate the effective microscopic densities, which are of interest for the present results, of the intercalated liquids.

The aim of this study is to elucidate the nature (i.e., how the molecules are actually moving) of the dynamics of the confined liquids. In order to achieve this we have used quasielastic neutron scattering (QENS) which makes it possible to study dynamics on different length scales and thereby to distinguish local, from nonlocal, e.g., long-range translational diffusive motion. Furthermore, since different dynamical processes occur on different time scales it is important to cover a wide dynamical range. This study is therefore carried out on three different QENS instruments: The highresolution backscattering spectrometer IN16 at ILL Grenoble France, ${ }^{40}$ the medium resolution inverted geometry backscattering spectrometer IRIS at ISIS Rutherford Appleton Laboratory $\mathrm{UK},{ }^{41}$ and the larger energy-transfer range timeof-flight spectrometer FOCUS at SINQ Paul Scherrer Institute Switzerland. The results from the IRIS measurements on the bulk and confined monomers and 7-mers have been published previously in Ref. 2 (and in Ref. 42, in the case of the bulk samples), and a preliminary analysis of all the present data was given in Ref. 6. However, in the former case it was difficult to obtain detailed information on the dynamics since the observed processes were only partly present in the limited experimental time window, and in Ref. 6 only the relaxation time averaged over all dynamical processes present in the experimental time window was considered.

\section{EXPERIMENT}

Vermiculite crystals (from Eucatex, Brazil) with an area of about $30 \mathrm{~mm}^{2}$ and a thickness of approximately $0.3 \mathrm{~mm}$ were washed and then treated for about a year with $1 M \mathrm{NaCl}$ solution at $50{ }^{\circ} \mathrm{C}$, with regular changes of solution, to produce a pure $\mathrm{Na}$-vermiculite. The $\mathrm{Na}$-vermiculite was thereafter dried in a vacuum furnace at $390 \mathrm{~K}$ for several days, before the clay was submerged in PG, 7-PG, and PPG, respectively. The clay samples were stored in this way for several weeks in order to allow the samples to reach full equilibrium, i.e., to let the maximum amounts of the liquids diffuse into the interlayer regions between the clay platelets. The prepared crystals were, after the surface liquid had been removed, weighed and then put directly into sample containers of aluminum.

As mentioned above the QENS measurements were performed on three instruments. IN16 (Ref. 40) was used with $\mathrm{Si}(111)$ analyzer and monochromator and an incident wavelength of $6.27 \AA$ with a total energy window of $\pm 15 \mu \mathrm{eV}$ and a full width at half maximum (FWHM) resolution of approximately $1 \mu \mathrm{eV}$. This setup gives a nearly Gaussianshaped resolution function, and the energy-transfer range is achieved by Doppler shifting the incident wavelength. The detectors were grouped into ten groups with $Q$ values in the range of $0.49-1.92 \AA^{-1}$. In the case of IRIS, ${ }^{41}$ we used PG002 analyzers and an analyzer wavelength of $6.67 \AA$ with a total energy window of $\pm 0.5 \mathrm{meV}$ and a resolution of $15 \mu \mathrm{eV}$. The detectors were grouped into ten groups with $Q$ values in the range of $0.51-1.82 \AA^{-1}$. Finally, the highenergy-transfer measurements (which were only performed on the bulk and confined PG and PPG samples) were carried out on FOCUS, where we used the PG002 monochromator and an incident wavelength of $3.99 \AA$ using an energy range up to $20 \mathrm{meV}$, in up-scattering. The detectors were grouped 
into eight groups with $Q$-elastic values in the range of $0.57-2.62 \AA^{-1}$. It should here be noted that the experimental data for a given $Q$ value were obtained at a constant scattering angle, and that this $Q$ then corresponds to the value in the case of elastic scattering, i.e., $Q=Q_{\text {elastic }}$. Furthermore, the obtained $S(Q, \omega)$ has a constant energy grid, which is necessary in order to be able to perform a Fourier transformation to the intermediate scattering function $S(Q, t)$ (see below).

By combining the three data sets a dynamical time window of 0.3-2000 ps was obtained. Since the total scattering of the samples is dominated by the large incoherent scattering cross section of hydrogen we essentially probe the selfdiffusion of the hydrogen. Thus, we were, to a good approximation, measuring the incoherent structure factor $S_{\text {inc }}(Q, \omega)$ [i.e., the time Fourier transform of the intermediate selfscattering function $\left.S_{\text {self }}(Q, t)\right]$ in our experiments.

The samples were orientated so that the clay platelets were at an angle of $135^{\circ}$ to the incident beam in order to make the elastic $Q$ vector parallel to the clay platelets for a scattering angle of $90^{\circ}$. This implies that the direction probed in the measurements is not the same for all scattering angles (i.e., $Q$ values). However, for most of the $Q$ values the component parallel to the clay layers strongly dominates (and the quasielastic contribution from motions perpendicular to the layers is expected to be rather low), which implies that we are mainly probing dynamics in the direction parallel to the clay layers. The used sample geometry makes also the data points at the highest $Q$ values less reliable due to the relatively high amount self-shielding of neutrons, which are scattered in the direction parallel to the clay platelets and sample container. In all measurements the samples were placed in flat $\mathrm{Al}$ containers with an internal thickness of $0.5 \mathrm{~mm}$. The actual thickness of the samples (i.e., the thickness of the vermiculite crystals containing the intercalated liquids) was in the range of $0.3-0.5 \mathrm{~mm}$. The elastic scattering from the empty containers was less than $5 \%$ of the total scattering from the samples. The corrections for absorption, background, and can scattering were performed using the ISIS standard program package IDA, ${ }^{43}$ also used for the subsequent analysis of the corrected data. The FOCUS data were corrected with another IDA package. ${ }^{44}$ A vanadium standard was used to measure the resolution function of each instrument. It should also be noted that only the scattering from the empty can was subtracted, and not the contribution from the clay platelets. Measurements were performed at the temperatures of 300 and $340 \mathrm{~K}$ on all instruments. Additional measurements at lower temperatures were performed on FOCUS and IN16, and measurements at $380 \mathrm{~K}$ were carried out on FOCUS and IRIS (there was no need to use the high resolution of IN16 at this high temperature since basically all the dynamics was faster than its dynamical time window).

\section{RESULTS AND DISCUSSION}

In our previous presentations of the results obtained on IRIS (Refs. 2 and 42) we observed, at least, three dynamical processes on a piconanosecond time scale at room temperature. Two of them were found to be weakly temperature dependent and $Q$ independent, where the faster one, with an

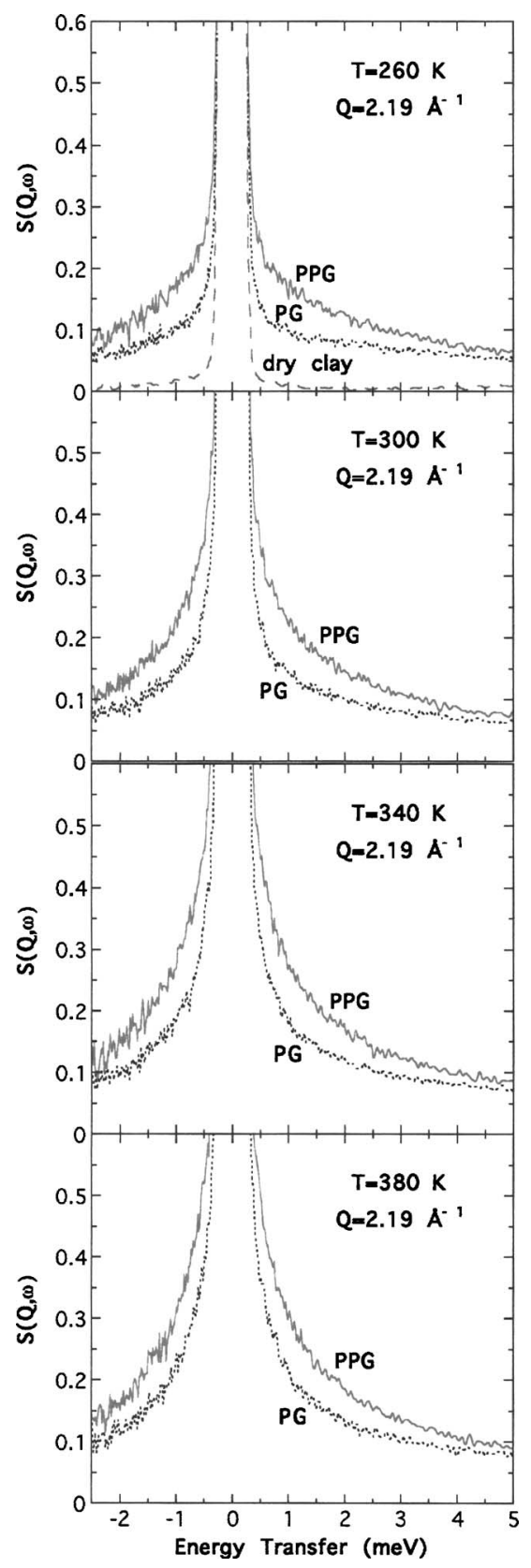

FIG. 1. Quasielastic neutron scattering spectra $S(Q, \omega)$ (in arbitrary units) taken on the FOCUS spectrometer for PG and PPG confined in $\mathrm{Na}-$ vermiculite clay. The four panels show constant scattering angle data for $Q_{\text {elastic }}=2.19 \AA^{-1}$ and the temperatures of $260,300,340$, and $380 \mathrm{~K}$, respectively. The data have been normalized so the total scattering is the same for both samples. In the top panel data for dry clay at $260 \mathrm{~K}$ are shown for comparison. It is almost equivalent to the experimental resolution function.

estimated correlation time of about 4 ps from the measurements on IRIS, arises from a local motion of hydrogen in the backbone of the monomers and the slower, by about a factor of ten, one is due to the methyl group rotation. ${ }^{2,42}$ The fast process is more evident from the FOCUS data shown in Fig. 1. It can be seen there that the quasielastic intensity of this local process is considerably stronger for confined PPG than for confined PG, particularly at low temperatures where the process is well separated from the other two processes. This is a clear result, which is independent of the exact normalization (e.g., a normalization to the elastic scattering would 


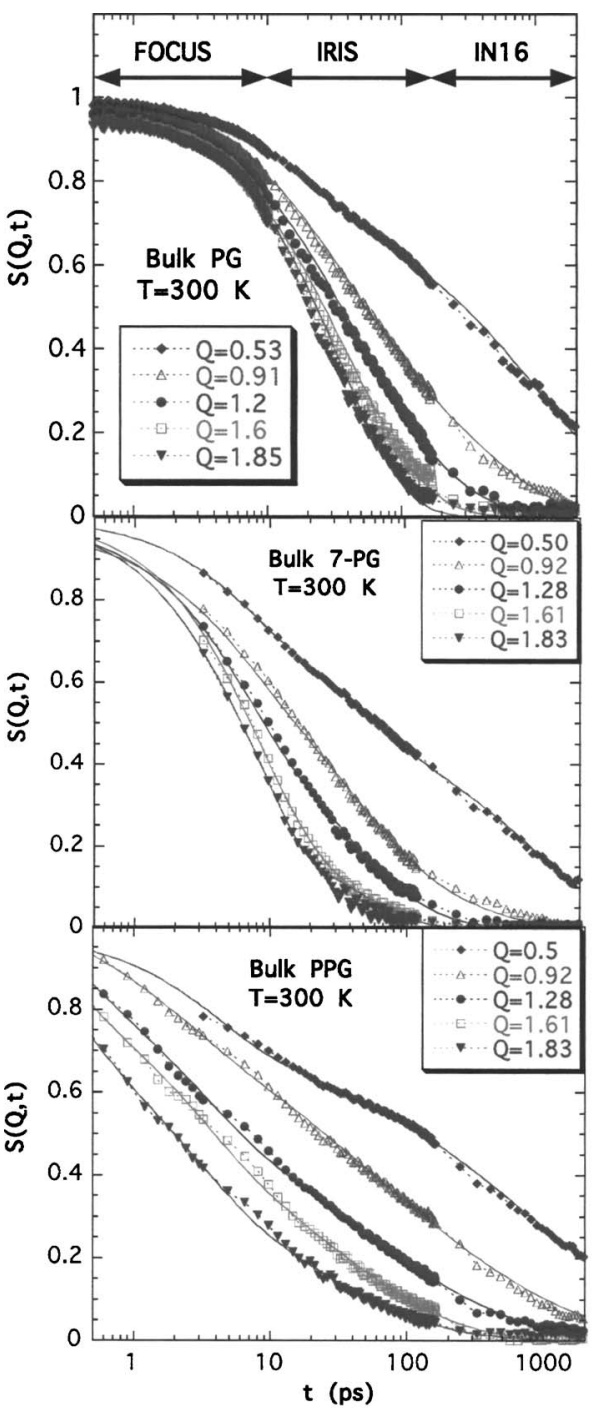

(a)

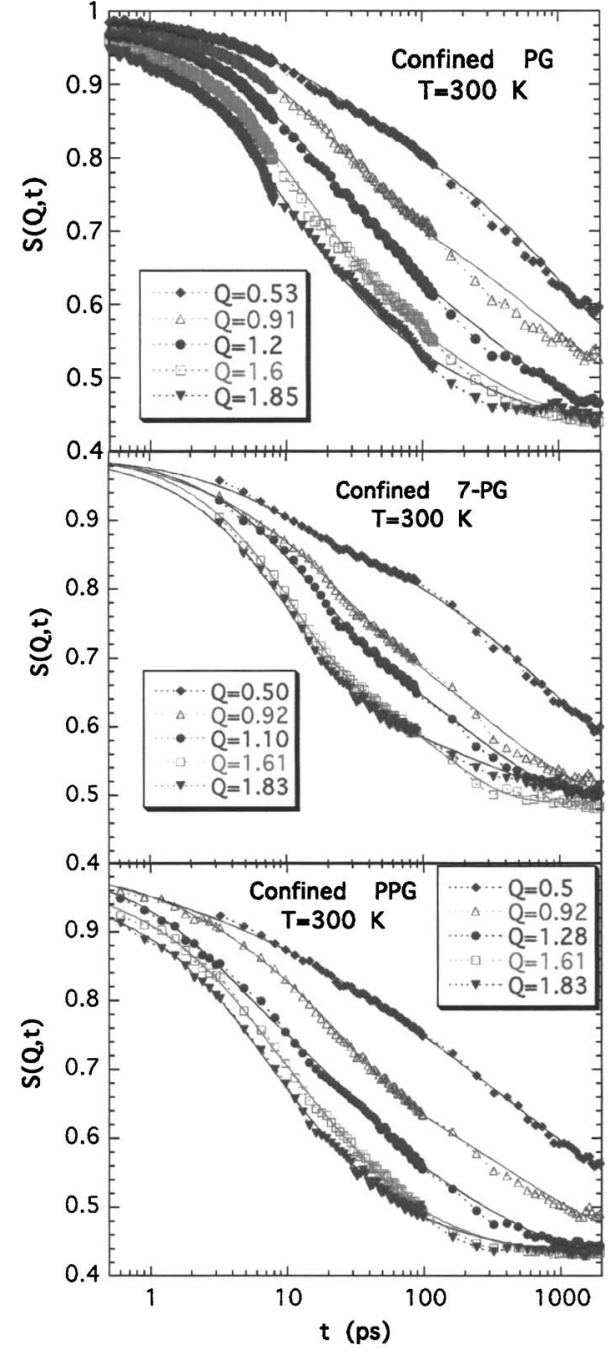

(b)
FIG. 2. Intermediate scattering functions $S(Q, t)$ at different $Q$ values for (a) bulk and (b) confined PG, 7-PG, and PPG at $T=300 \mathrm{~K} . S(Q, t)$ is combined from data taken on FOCUS, IRIS, and IN16, as indicated in (a). The experimental data have been fitted by three different relaxation functions (see the text). give the same conclusion as the present normalization to the total scattering). Thus, this local motion of hydrogen increases when the monomers are connected to polymer chains (this effect is even larger for the bulk samples). Since the third process, which describes the more long-range translational or segmental motion of single or connected monomers, shows a strong $Q$ dependence and nonexponential relaxation it cannot be covered in the dynamic time window of a single QENS instrument. Therefore, the measured $S(\mathrm{Q}, \omega)$ on the three different instruments were Fourier transformed to intermediate scattering functions $S(Q, t)$ covering different time ranges. These functions were thereafter combined into a single relaxation function over the total dynamic time window of $0.3-2000$ ps (when data from all the three instruments were used). At room temperature the three dynamic processes were then almost entirely covered in this wide time window. This is shown in Fig. 2, which shows such combined $S(Q, t)$ for bulk and confined PG, 7-PG, and PPG at $T=300 \mathrm{~K}$.

In this paper we have tried to separate the segmental and/or translational dynamics from the methyl group rotation and the fast local dynamics shown in Fig. 1. Thus, at $T$
$=300 \mathrm{~K}$ the data shown in Fig. 2 have been described by three Kohlrausch-Williams-Watts (KWW)-stretched exponential relaxation functions 45,46

$$
\begin{aligned}
S(Q, t)= & A_{1} \exp \left[-\left(\frac{t}{\tau_{f}}\right)\right]+A_{2} \exp \left[-\left(\frac{t}{\tau_{r}}\right)^{0.9}\right] \\
& +\left(1-A_{1}-A_{2}-B\right) \exp \left[-\left(\frac{t}{\tau_{d}}\right)^{\beta_{\mathrm{KWw}}}\right]+B, \\
& 0<\beta_{\mathrm{KWW}}<1,
\end{aligned}
$$

where $\tau_{f}, \tau_{r}$, and $\tau_{d}$ are typical relaxation times for fast local motions, the methyl group rotation, and the diffusive process, respectively. $\beta_{\mathrm{KWW}}$ is a stretching parameter, which was kept fixed to 1 (i.e., a single exponential function) in the case of the fast local process and to 0.9 (in accordance with results reported in Ref. 47) for the methyl group rotation. $A_{1}$ and $A_{2}$ are the amplitudes of the fast local motions and the methyl group rotation, respectively, and $B$ is the long time limit of the three relaxation functions, which is zero for our bulk samples and about 0.45 for the clay samples where we have immobile hydrogen in the clay platelets (a slight $Q$ dependence of $B$ exists due to the Debye-Waller factor of 
these immobile $\mathrm{H}$ atoms). In the case of the methyl group rotation we fixed the correlation time to $\tau_{r}=44 \mathrm{ps}$ for the bulk samples and $\tau_{r}=20 \mathrm{ps}$ for the confined samples, as these were the approximate values obtained from the IRIS experiment. ${ }^{2,48}$ In the IRIS experiment the fast local motion was estimated to be about $\tau_{f}=4 \mathrm{ps}$ for all samples and $Q$ values, however, this process is more accurately determined in the FOCUS experiment (due to its higher maximum energy transfer) and therefore we did not keep this value fixed in the fitting procedure, which gave values in the range of $0.8-4 \mathrm{ps}$ for this process. Thus, in total we used six free fit parameters in the fitting procedure. The remaining third process (which, in fact, may include more than one dynamical process) is rather stretched, with $\beta_{\mathrm{KWW}}=0.55 \pm 0.15$ for confined PG and 7-PG, $0.65 \pm 0.15$ for bulk PG and 7-PG, and $0.4 \pm 0.1$ for bulk and confined PPG. Thus, the stretching slightly increases with increasing chain length, particularly for the bulk samples, and there is no clear $Q$ dependence. For the monomer and 7-mer $\beta_{\mathrm{KWW}}$ tends to be slightly lower in confinement [although the difference is within the error bars of each $S(Q, t)$ ], as also observed in NMR (Ref. 9) and dielectric experiments ${ }^{8}$ as well as molecular-dynamics (MD) simulations ${ }^{10}$ of polymers intercalated in other types of natural or synthetic clays. However, in the case of PPG there was no indication of any decrease of $\beta_{\mathrm{KWW}}$ in confinement.

For most temperatures and $Q$ values it is also possible to obtain a reasonable fit with a single KWW function, and that was what we had to use for the other temperatures $(260,340$, and $380 \mathrm{~K})$ for which we were not able to separate the correlation times for the fast local motion and the methyl group rotation from the translational or segmental motion. Thus, at these temperatures we obtain a relaxation time representing a weighted average value of the three processes. Such a simplification has the limitation that it does not account for the fact that the weighting factors of the processes depend on both $Q$ and temperature. Nevertheless, a rough estimate of how this average relaxation time $\langle\tau\rangle$ varies with $Q$ and temperature can be obtained for the different samples.

In Fig. 3(a) we compare $S(Q, t)$ at $T=300 \mathrm{~K}$ and $Q$ $=0.92 \AA^{-1}$ for all the investigated samples. In the case of the confined liquids, the elastic contribution from the clay platelets has been removed in order to more easily compare the relaxation functions for the confined and bulk liquids, i.e., the long time limit of $S(Q, t)$, estimated to 0.43 for confined PG and PPG and 0.49 for 7-PG, was first subtracted and thereafter $S(Q, t)$ was appropriately renormalized. We note that the confinement always slows down the system (the filled symbols are always below the empty symbols). Some of the previous findings are evident again, for instance, that the fast local motion (including the Debye-Waller factor) increases with increasing chain length (in the region below 5 ps the longer chains have relaxed more) and reduces in confinement, the exception being the monomer where this fast local motion is almost absent from the beginning. The monomer PG and PPG are influenced differently. For PPG some of the fast relaxation shifts to longer times when confined but at longer times the influence is small. The opposite is true for PG, where the short time behavior is hardly changed at all but at longer times the dynamics is slowed down by the

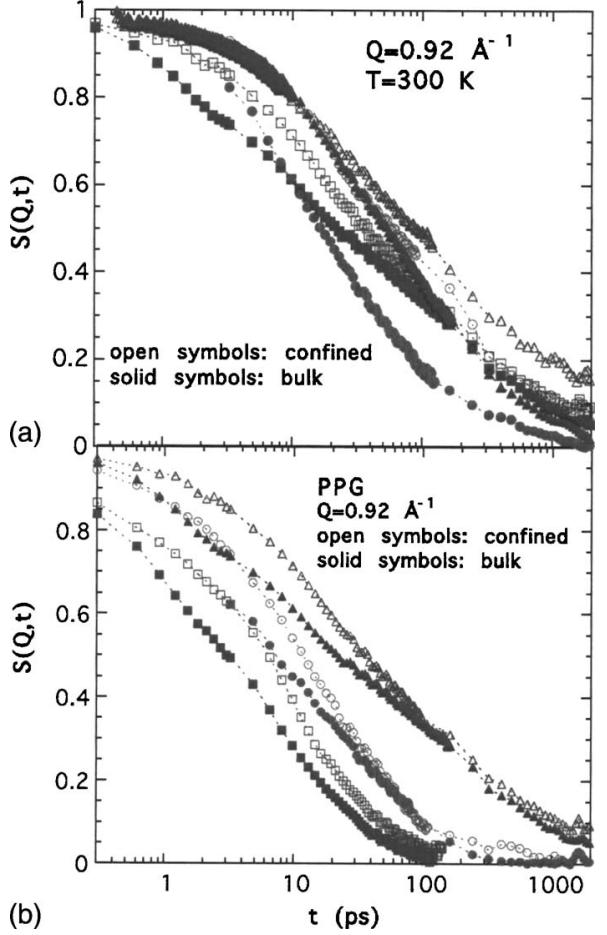

FIG. 3. (a) Intermediate scattering functions $S(Q, t)$ for bulk and confined PG $(\Delta), 7-\mathrm{PG}(\mathrm{O})$, and PPG( $\square)$ at $T=300 \mathrm{~K}$ and $Q=0.92 \AA^{-1}$. (b) $S(Q, t)$ for bulk and confined PPG at $Q=0.92 \AA^{-1}$ and the temperatures of $300(\Delta)$, $340(\mathrm{O})$, and $380 \mathrm{~K}(\square)$.

confinement. The reason for this is to be sought in the nature of the motion. There is almost no local short time backbone motion to be slowed down in PG, simply because there is basically no backbone as such. The long-range diffusion, at longer times, will, however, be slowed down by the geometrical confinement. For PPG between 100 and 1000 ps it does not seem to matter much that the motion is confined to 2D. This indicates that the dynamics at these times are already restricted in the bulk by the chain connectivity. Let us also investigate how the relaxation rate is affected by the temperature. Figure 3(b) shows $S(Q, t)$ for confined and bulk PPG at $Q=0.92 \AA^{-1}$ and the temperatures of 300,340 , and $380 \mathrm{~K}$. The figure shows, once again, that the 2D confinement present slows down the fast process. It is also evident, as expected, that the relaxation rate increases significantly with increasing temperature. Similar findings are obtained for the monomers and 7-mers.

Let us now elucidate the $Q$ dependence of the third relaxation process, due to translational and/or segmental motions, in more detail. The average relaxation time of this process, which was obtained from the KWW fits shown in Fig. 2 through the relation ${ }^{49}$

$$
\left\langle\tau_{d}\right\rangle=\frac{\tau_{d}}{\beta_{\mathrm{KWW}}} \Gamma\left(\frac{1}{\beta_{\mathrm{KWW}}}\right),
$$

where $\Gamma$ is the gamma function, shows an interesting chainlength dependence. In the clay the relaxation rate is almost independent of the chain length, whereas a strong chainlength dependence is observed for the bulk samples. This is shown in Fig. 4, which shows the $Q$ dependence of the average relaxation time of this third process, plotted as $1 /\left\langle\tau_{d}\right\rangle$ 


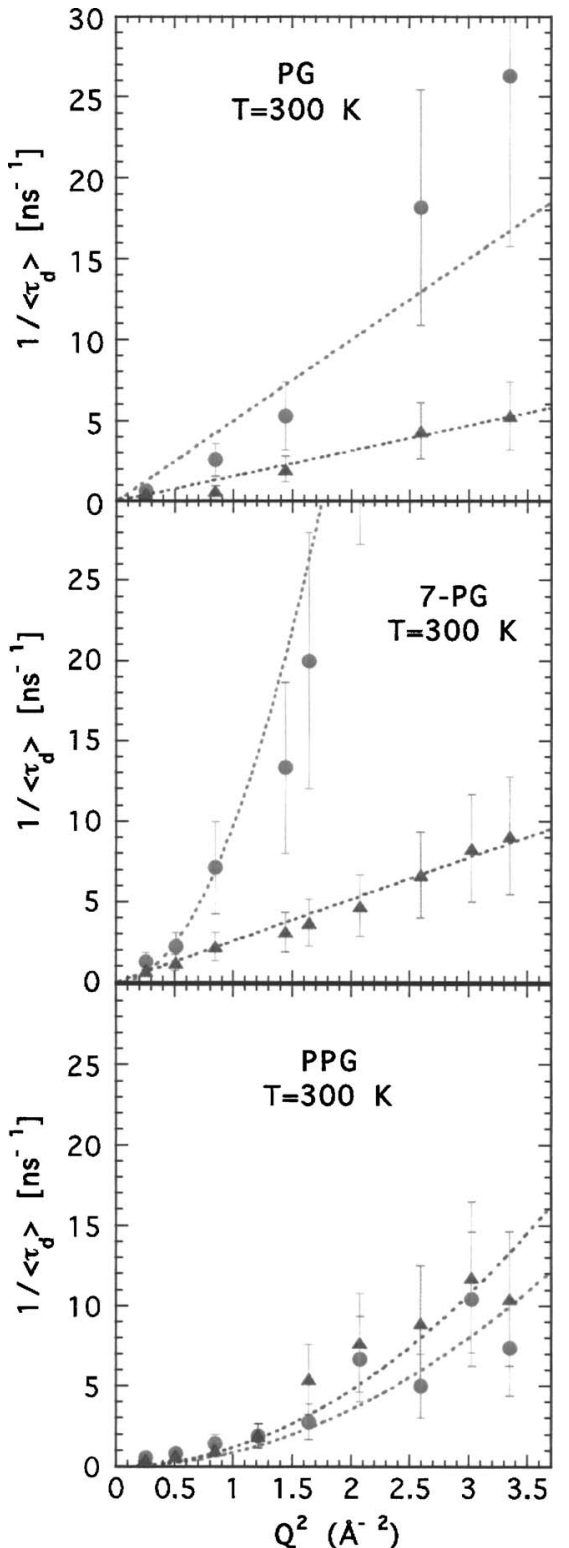

FIG. 4. $1 /\left\langle\tau_{d}\right\rangle$ as a function of $Q^{2}$, for PG, 7-PG, and PPG in bulk (O) and confined in clay $(\Delta)$. The $Q$ dependence of $1 /\left\langle\tau_{d}\right\rangle$ has been fitted by a $Q^{\nu}$ function (given by the lines) in the case of 7-PG and PPG, whereas for PG, where ordinary translational diffusion is expected [i.e., no Rouse dynamics (Refs. 51 and 52) as for the longer chains], a $Q^{2}$ dependence is indicated. From this fitting procedure an approximate $Q^{2.6}$ dependence of $1 /\left\langle\tau_{d}\right\rangle$ is obtained for confined and bulk PPG, suggesting a mixture of Rouse dynamics (Refs. 52 and 53) and segmental motions.

as a function of $Q^{2}$, for PG, 7-PG, and PPG in bulk as well as confined in clay. As can be seen in Fig. 4 the behaviors in bulk and clay are very different. In the bulk this third process is considerably faster for the 7-mer compared to the monomer and polymer [which is even clear from a direct inspection of the data shown in Figs. 2 and 3(a)]. The reason for this "anomalous" chain-length dependence is that the slow dynamics of oligomers tends to slow down with increasing chain length. This is a general behavior that has been observed for all kinds of oligomers, including oligomers of PG of a few monomer units or more. However, the monomer of PG does not follow this trend, which implies that its main relaxation should be faster than for the longer oligomers, due to its tendency to form hydrogen bonds to neighboring monomers. In this way the monomers are connected to a hydrogen-bonded network, which reduces both the relaxation rate and the fragility of the liquid. ${ }^{42,50}$ Nevertheless, the dynamics of bulk PG is faster than for confined PG. On the other hand, if the formation of a three-dimensional hydrogen-bonded network had not been prevented in the clay, the difference would have likely been even larger as indicated by the large difference between bulk and confined 7-PG [see Fig. 4(b)]. However, for PPG there is no difference (within the error bars) between the bulk and confined samples. The strong chain-length dependence in bulk and the almost absent chain-length dependence in the 2D confinement can be understood by considering the physical nature of this diffusive process. In the clay the motions have, of necessity, to be of a two-dimensional character, whereas in bulk the dimensionality of the diffusive motions is likely to be chain-length dependent. The monomers should have approximately the same ability to move in all three directions, whereas the motion of polymer segments should be, at least partly, prevented by surrounding polymer chains. Thus, the diffusive motion of polymer segments should be more restricted and has a low dimensional character, similar to the 2D geometry in the clay. This is the most likely explanation as to why the bulk dynamics approaches that in the clay for the longest chains.

In Fig. 4 it is further seen that the inverse of the relaxation time is proportional to $Q^{\nu}$, where $2 \leqslant \nu \leqslant 3$. In the case of monomers and short chains we expect to have $Q^{2}$ dependence, typical for ordinary translational diffusion, whereas for the longer chains this process is likely to be more complex with a transition to so-called Rouse dynamics ${ }^{51,52}$ ( $\nu$ =4) at $Q \leqslant 0.2 \AA^{-1} \cdot{ }^{53}$ This is also observed for our bulk and confined samples of PPG, which exhibit $\nu$ values of about 2.6. A similar good agreement with the expected $Q^{2}$ dependence is not obtained for the PG samples and bulk 7-PG, where the data points indicate a slightly stronger $Q$ dependence than is expected for ordinary diffusion. However, one should note that the error bars (mainly caused by the separation procedure of the three processes) are so large that it is not possible to unambiguously distinguish between, for instance, $Q^{2}$ and $Q^{3}$ dependences. Thus, Fig. 4 is only providing a rough estimate of the $Q$ dependence of this relaxation process.

As mentioned above, we have not been able to separate the three dynamical processes at other temperatures than 300 $\mathrm{K}$, so in order to describe the temperature dependence of the QENS data we have to do it for the weighted average value for the three processes [as in Fig. 3(b) where the total relaxation function was described by a single KWW function]. If we estimate this average relaxation time from neutron scattering at $Q=0.92 \AA^{-1}$ we are furthermore able to make a rough comparison with dielectric results, ${ }^{3,4}$ since approximately the same $Q$ value seems to be probed by dielectric spectroscopy. ${ }^{54}$ Figure 5 shows the temperature dependence of the average relaxation time from neutron scattering as well as the dielectric $\alpha$ relaxation time for PG (Ref. 4) and PPG (Ref. 3) in bulk and confined in clay. The agreement is unexpectedly good considering that the neutron data contain 


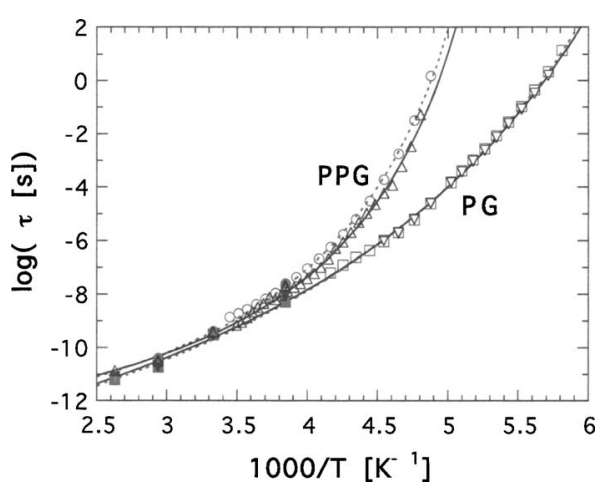

FIG. 5. Temperature dependence of the average relaxation times for PG ( $\square$ ) and PPG $(\mathrm{O})$ in bulk and confined in clay ( $\nabla$ and $\Delta$, respectively). The dielectric $\alpha$ relaxation is given by the open symbols whereas the average relaxation time from neutron scattering is marked with the filled symbols. Note the excellent agreement between the average relaxation time from neutron scattering and the dielectric $\alpha$ relaxation time, evident from the simultaneous fitting with the Vogel-Fulcher-Tamman (Ref. 55) (VFT) equation (the dashed line for bulk and the solid line for confined liquid).

all kinds of hydrogen motions on the experimental time scale and not only motions related to the dielectric $\alpha$ relaxation. On the other hand, at these relatively high temperatures the dielectric $\alpha$ and $\beta$ relaxations are merged, which means that the dielectric data should also give an average relaxation time of most dynamics on a length scale corresponding to approximately $Q=1 \AA^{-1}$. Thus, although different dynamics is probed by neutron scattering and dielectric spectroscopy a reasonable agreement is perhaps not totally unexpected. In Fig. 5 it can furthermore be seen that the difference in $\left\langle\tau_{d}\right\rangle$ between the samples (see Fig. 4) is now reduced when an averaging of all $\mathrm{H}$ atom dynamics is taken (the differences also look smaller on the log scale of Fig. 5). The good agreement between the neutron data and dielectric data makes it possible to describe the temperature dependence of both relaxation times of each sample with the same Vogel-FulcherTamman (VFT) function ${ }^{55}$

$$
\tau=\tau_{0} \exp \left(\frac{D T_{0}}{T-T_{0}}\right),
$$

where $D$ is a material-dependent constant describing the degree of non-Arrhenius behavior, $T_{0}$ is the temperature where $\tau$ goes to infinity, and $\tau_{0}$ is the relaxation time extrapolated to infinite temperature, which usually corresponds to quasilattice and molecular vibrations of the order of $10^{-14} \mathrm{~s}$. This fact is evident in Fig. 5, where it can be further seen that the present confinement has a relatively small influence, compared to other types of confinements, on the overall dynamics probed by QENS and dielectric spectroscopy. The parameters from the VFT fits and the estimated glass transition temperatures $T_{g}$, based on the temperature where the relaxation times are $100 \mathrm{~s}$, are given in Table I. For the bulk samples there is a good agreement with values reported in the literature (e.g., with Ref. 19, as shown in Table I). In the case of our two-dimensional confined liquids with a thickness of a single molecular layer it is difficult to make a quantitative comparison with results available in the literature because either the confined liquid or the geometry of the confined space is distinctly different or the layer thickness is
TABLE I. Estimated VFT parameters and glass transition temperatures (defined as the temperature for which $\tau \approx 100 \mathrm{~s}$ ) from the fits shown in Fig. 5.

\begin{tabular}{lcccc}
\hline \hline Sample & $\tau_{0}(\mathrm{fs})$ & $D$ & $T_{0}(\mathrm{k})$ & $T_{g}(\mathrm{~K})$ \\
\hline Bulk PG & 2.5 & 19.3 & 111.9 & 168.8 \\
Bulk PG (Ref. 19) & & 18.4 & 112.3 & 166.8 \\
Confined PG & 2.0 & 19.1 & 112.8 & 168.8 \\
Bulk PPG & 72 & 6.9 & 165.3 & 199.8 \\
Bulk PPG (Ref. 19) & & 7.0 & 164.6 & 197.4 \\
Confined PPG & 37 & 7.7 & 164.0 & 198.4 \\
\hline \hline
\end{tabular}

considerably larger. Nevertheless it is interesting to discuss the present results in relation to findings from MD simulations of confined model systems. Baschnagel and Binder ${ }^{32}$ studied polymer melts confined between two parallel noninteracting smooth hard walls and they found that the diffusion coefficient in a direction parallel to the walls was almost indistinguishable from that of the bulk. On the other hand, if we instead have a liquid-hard wall interface then both an attractive force to the walls (provided that the thermal energy is lower than the attraction energy) and rough surfaces are likely to slow down the dynamics. ${ }^{26}$ This is supported by MD simulations of simple diatomic liquids confined between two parallel smooth interacting walls ${ }^{20}$ or in a pore surrounded by the same, but frozen, liquid. ${ }^{33}$ In both studies the dynamics was dramatically slowed down close to the walls due to surface interactions in the former case and the rough interface in the latter case. Thus, provided that these results are also relevant for our confined liquids, it seems likely that the clay surfaces are rather smooth and that their interaction with the intercalated liquids is weak (at least for PG and its oligomers).

The present findings are interesting since, for instance, the relaxation time of the dielectric $\alpha$ process, which is generally accepted to reflect the cooperative motions in the material, is expected to be affected by both the confinement to a single molecular layer and the interactions with the clay surfaces. A possible explanation is, of course, that the two effects cancel out by working in opposite directions, although that appears to be a very improbable coincidence to occur for both PG and PPG, since it is generally found (see, e.g., Ref. 28) that these effects do not cancel out at all temperatures. The reason for this is that the influence of surface interactions and spatial confinement effects are very different at different temperatures (the speeding up due to spatial confinement dominates at low temperatures, whereas the slowing down due to surface interactions dominates at high temperatures), giving rise to different temperature dependences of the $\alpha$ relaxation in confinement and bulk. Furthermore, Alba-Simionesco et al. ${ }^{29}$ have shown that if both these effects are strong the glass transition region tends to be very broad (at least in the case when there are several molecular layers of the confined liquid), i.e., the $\alpha$ relaxation is expected to be much broader in confinement, which neither seems to be a significant finding in the present study. Thus, in contrast with what one would expect this suggests that both the present confinement, where the motion of the intercalated liquids is restricted in only one dimension, and the 
interactions with the clay surfaces have a relatively small influence on the molecular dynamics probed in this study. A possible explanation for the generally weak geometrical confinement effect may be that the cooperativity length is not affected by the extreme confinement in the direction perpendicular to the clay platelets. If the cooperatively rearranging region in a bulk material has a two-dimensional character then one would not expect that the cooperativity length would be strongly affected by confinement as long as there are no restrictions for motions in two dimensions. The weak interaction with the clay surfaces, for all the chain lengths, indicates that the $\mathrm{OH}$ end groups do not form strong hydrogen bonds to the clay surfaces. Rather, it seems as the surface interactions are considerably weaker than in many other model systems (e.g., unmodified Vycor glasses and zeolites) commonly used to confine liquids and polymers.

\section{CONCLUSION}

The present QENS study of PG and two of its oligomers confined in a $\mathrm{Na}$-vermiculite clay supports previous dielectric results on the same systems and shows that most of the relaxational dynamics is only weakly affected by the confinement. Since the intercalated liquids are confined to a single molecular layer, which means that all the molecules are only able to move in two dimensions and should in some way interact with the clay surfaces, it is interesting and unexpected that both the pure geometrical confinement effect and the surface interactions appear to be very weak. The most pronounced difference is observed for the slow translational diffusion of short oligomers, which is slowed down in the clay resulting in a reduced chain-length dependence compared to bulk. This fact can be understood by considering the physical nature of this diffusive process. In the clay the motions have, of necessity, to be of a two dimensional character, whereas in bulk the dimensionality of the diffusive motions is likely to be chain-length dependent. The monomers should have approximately the same ability to move in all three directions, whereas the motion of polymer segments should be, at least partly, prevented by surrounding polymer chains. Thus, the diffusive motion of polymer segments should be more restricted and has a low dimensional character, similar to the $2 \mathrm{D}$ geometry in the clay. This is the most likely explanation as to why the bulk dynamics approaches that in the clay for the longest chains.

\section{ACKNOWLEDGMENTS}

One of the authors (J.S.) is a Royal Swedish Academy of Sciences Research Fellow supported by a grant from the Knut and Alice Wallenberg Foundation. The Swedish Research Council and Swedish Foundation for Strategic Research are acknowledged for financial support. A part of the work was done at SINQ, PSI.

\footnotetext{
${ }^{1}$ R. Bergman and J. Swenson, Nature (London) 403, 283 (2000).

${ }^{2}$ J. Swenson and W. S. Howells, J. Chem. Phys. 117, 857 (2002)

${ }^{3}$ G. A. Schwartz, R. Bergman, J. Mattsson, and J. Swenson, J. Chem. Phys. 120, 5736 (2004)

${ }^{4}$ R. Bergman, J. Mattsson, C. Svanberg, G. A. Schwartz, and J. Swenson, Europhys. Lett. 64, 675 (2003).
}

${ }^{5}$ S. Cerveny, J. Mattsson, J. Swenson, and R. Bergman, J. Phys. Chem. B 108, 11596 (2004).

${ }^{6}$ J. Swenson, G. A. Schwartz, R. Bergman, and W. S. Howells, Eur. Phys. J. E 12, 179 (2003).

${ }^{7}$ E. P. Giannelis, R. Krishnamoorti, and E. Manias, Adv. Polym. Sci. 138, 107 (1999).

${ }^{8}$ S. H. Anastasiadis, K. Karatasos, G. Vlachos, E. Manias, and E. P. Giannelis, Phys. Rev. Lett. 84, 915 (2000).

${ }^{9}$ D. B. Zax, D.-K. Yang, R. A. Santos, H. Hegemann, E. P. Giannelis, and E. Manias, J. Chem. Phys. 112, 2945 (2000).

${ }^{10}$ E. Manias, V. Kuppa, D.-K. Yang, and D. B. Zax, Colloids Surf., A 187-188, 509 (2001).

${ }^{11}$ J. J. Tuck, P. L. Hall, M. H. B. Hayes, D. K. Ross, and C. Poinsignon, J. Chem. Soc., Faraday Trans. 1 80, 309 (1984).

${ }^{12}$ D. J. Cebula, R. K. Thomas, and J. W. White, Clays Clay Miner. 29, 241 (1981).

${ }^{13}$ N. T. Skipper, A. K. Soper, and D. C. McConnell, J. Chem. Phys. 94, 5751 (1991)

${ }^{14}$ R. K. Hawkins and P. A. Egelstaff, Clays Clay Miner. 28, 19 (1980).

${ }^{15}$ S. Kralj, A. Zidansek, G. Lahajnar, I. Musevic, S. Zumer, R. Blinc, and M. M. Pintar, Phys. Rev. E 53, 3629 (1996).

${ }^{16}$ S. Stapf, R. Kimmish, and R.-O. Seitter, Phys. Rev. Lett. 75, 2855 (1995).

${ }^{17}$ G. Schwalb and F. W. Deeg, Phys. Rev. Lett. 74, 1383 (1995).

${ }^{18}$ J. Schüller, Yu. B. Melnichenko, R. Richert, and E. W. Fischer, Phys. Rev. Lett. 73, 2224 (1994).

${ }^{19}$ Y. B. Melnichenko, J. Schuller, R. Richert, B. Ewen, and C. K. Loong, J. Chem. Phys. 103, 2016 (1995).

${ }^{20}$ R. Yamamoto and K. Kim, J. Phys. IV 10, 15 (2000).

${ }^{21}$ M. Arndt, R. Stanmarius, H. Groothues, E. Hempel, and F. Kremer, Phys. Rev. Lett. 79, 2077 (1997).

${ }^{22}$ G. Barut, P. Pissis, R. Pelster, and G. Nimtz, Phys. Rev. Lett. 80, 3543 (1998).

${ }^{23}$ A. Schönhals and R. Stauga, J. Chem. Phys. 108, 5130 (1998).

${ }^{24}$ A. Schönhals and R. Stauga, J. Non-Cryst. Solids 235-237, 450 (1998).

${ }^{25}$ A. Huwe, F. Kremer, P. Behrens, and W. Schwieger, Phys. Rev. Lett. 82, 2338 (1999)

${ }^{26}$ J. Baschnagel, C. Mischler, and K. Binder, J. Phys. IV 10, 9 (2000).

${ }^{27}$ A. Huwe, F. Kremer, L. Hartmann et al., J. Phys. IV 10, 59 (2000).

${ }^{28}$ A. Schönhals, H. Goering, Ch. Schick, B. Frick, and R. Zorn, Eur. Phys. J. E 12, 173 (2003).

${ }^{29}$ C. Alba-Simionesco, G. Dosseh, E. Dumont, B. Frick, B. Geil, D. Morineau, V. Teboul, and Y. Xia, Eur. Phys. J. E 12, 19 (2003).

${ }^{30}$ R. Pelster, Phys. Rev. B 59, 9214 (1999).

${ }^{31}$ V. Crupi, D. Majolino, P. Migliardo, and V. Venuti, J. Phys. Chem. A 104, 11000 (2000)

${ }^{32}$ J. Baschnagel and K. Binder, Mater. Res. Soc. Symp. Proc. 543, 157 (1999).

${ }^{33}$ P. Scheidler, W. Kob, and K. Binder, Europhys. Lett. 52, 277 (2000).

${ }^{34}$ W. Gorbatschow, M. Arndt, R. Stannarius, and F. Kremer, Europhys. Lett. 35, 719 (1996)

${ }^{35}$ B. Böddeker and H. Teichler, Phys. Rev. E 59, 1948 (1999).

${ }^{36}$ A. L. Demirel and S. Granick, Phys. Rev. Lett. 77, 2261 (1996).

${ }^{37}$ T. Fehr and H. Löwen, Phys. Rev. E 52, 4016 (1995).

${ }^{38}$ D. Morineau, Y. Xia, and C. Alba-Simionesco, J. Chem. Phys. 117, 8966 (2002).

${ }^{39}$ G. B. McKenna, J. Phys. IV 10, 53 (2000); Eur. Phys. J. E 12, 191 (2003).

${ }^{40}$ B. Frick and M. Gonzalez, Physica B 301, 8 (2001).

${ }^{41}$ C. J. Carlie and M. A. Adams, Physica B 182, 431 (1992).

${ }^{42}$ J. Swenson, I. Köper, and M. T. F. Telling, J. Chem. Phys. 116, 5073 (2002).

${ }^{43}$ W. S. Howells, Technical Report Rutherford Appleton Laboratory, Report No. RAL-TR 96,006, 1996

${ }^{44} \mathrm{~J}$. Wuttke, http://www.e13.physik.tu-muenchen.de/Wuttke/Ida.html

${ }^{45}$ R. Kohlrausch, Ann. Phys. Chem. 72, 383 (1847).

${ }^{46}$ G. Williams and D. C. Watts, Trans. Faraday Soc. 66, 80 (1970).

${ }^{47}$ C. Saelee, T. M. Nicholson, and R. G. Davies, Macromolecules 33, 2258 (2000).

${ }^{48}$ D. Andersson, P. Carlsson, L. M. Torell, L. Börjesson, R. L. McGreevy, and W. S. Howells, Physica B 266, 126 (1999). 
${ }^{49}$ C. P. Lindsey and G. D. Patterson, J. Chem. Phys. 73, 3348 (1980).

${ }^{50}$ R. Bergman, C. Svanberg, D. Andersson, A. Brodin, and L. M. Torell, J. Non-Cryst. Solids 235-237, 225 (1998).

${ }^{51}$ P. E. Rouse, J. Chem. Phys. 21, 1272 (1953).

${ }^{52}$ M. Doi, Introduction to Polymer Physics (Oxford University Press, Oxford, UK, 1995).
${ }^{53}$ D. Andersson, D. Engberg, R. Zorn, and L. Börjesson (unpublished).

${ }^{54}$ A. Arbe, A. Alegria, J. Colmenero, S. Hoffmann, L. Willner, and D. Richter, Macromolecules 32, 7572 (1999).

${ }^{55}$ H. Vogel. Phys. Z. 22, 645 (1921); G. S. Fulcher, J. Am. Chem. Soc. 8, 339 (1925); G. Tammann and G. Hesse, Z. Anorg. Allg. Chem. 156, 245 (1926). 